\title{
A Cross-sectional Examination of the Clinical Significance of Autistic Traits in Individuals
}

\section{Experiencing a First Episode of Psychosis}

Katharine Chisholm*a,b Mirabel Pelton ${ }^{\text {b,c }}$, Nikita Duncan ${ }^{\mathrm{b}}$, Katherine Kidd ${ }^{\mathrm{b}}$, Klaas J. Wardenaar ${ }^{\mathrm{d}}$, Rachel Upthegrove ${ }^{\mathrm{b}}$, Matthew R Broome ${ }^{\mathrm{b}, \mathrm{f}}$, Ashleigh Lin ${ }^{\mathrm{g} \wedge}$, Stephen J Wood ${ }^{\mathrm{b}, \mathrm{h} \wedge}$

${ }^{a}$ Department of Psychology, Aston University, UK

b School of Psychology and Institute for Mental Health, University of Birmingham, UK

${ }^{\mathrm{c}}$ Centre for Innovative Research Across the Life course (CIRAL), Coventry University, UK

${ }^{d}$ Department of Psychiatry, Interdisciplinary Center Psychopathology and Emotion Regulation (ICPE), University of Groningen, University Medical Center Groningen, The Netherlands

e School of Psychiatry, Institute of Clinical Sciences, and Institute for Mental Health, University of Birmingham. Forward Thinking Birmingham and Birmingham and Solihull Mental Health Foundation Trust

${ }^{\mathrm{f}}$ Department of Psychiatry, University of Oxford, UK; Faculty of Philosophy, University of Oxford, UK; Oxford Health NHS Foundation Trust, UK.

${ }^{g}$ Telethon Kids Institute, The University of Western Australia

${ }^{\mathrm{h}}$ Orygen, The National Centre of Excellence for Youth Mental Health, Melbourne, Australia, \& the Centre for Youth Mental Health, University of Melbourne, Australia, \& School of Psychology, University of Birmingham, UK

^joint last author

* Corresponding author: Katharine Chisholm, Department of Psychology, Aston University, UK, UK, 0121204 4169, k.chisholm@aston.ac.uk

Declarations of interest: none 


\begin{abstract}
Autism traits are found at elevated rates in individuals with schizophrenia spectrum disorders, however, there is a lack of evidence regarding potential clinical impact. The current research aimed to examine potential associations between autism traits and symptoms of psychosis, social and role functioning, and quality of life. 99 individuals experiencing a first episode of psychosis took part in a cross-sectional interview and self-report questionnaire which assessed current symptoms of psychosis, autism traits, functioning, and quality of life. Participants were found to have a high level of autism traits. Higher autism traits were associated with poorer quality of life, functioning, and current psychotic symptoms. Receiver operating characteristic curve (ROC) analyses indicated that optimal AQ cut-off scores to predict severity of psychosis symptoms, functioning, and quality of life were lower than those used to suggest likely autism-spectrum diagnosis. Results suggest that autism traits are associated with poorer clinical presentation in first-episode psychosis populations, even in those whose traits fall below potentially diagnostic thresholds for autism. Psychosis services should be prepared to adequately address the needs of individuals with higher autism traits.
\end{abstract}

\title{
Keywords:
}

Autism traits; Functioning; Quality of life; Schizophrenia spectrum disorders 


\section{A Cross-sectional Examination of the Clinical Significance of Autistic Traits in Individuals Experiencing a First Episode of Psychosis}

\section{Introduction}

Autism spectrum conditions (ASC) and schizophrenia spectrum disorders (SSD) are reported to co-occur at elevated rates (Davidson et al., 2014; Hofvander et al., 2009), and have been found to share many phenotypic and neurological similarities, and multiple risk factors (Carroll \& Owen, 2009; Cheung et al., 2010; Chisholm et al, 2015). As well as potentially shedding light on the fundamental aetiology of the conditions, understanding the similarities and differences between the conditions and the extent of the overlap may also aid with the development of specialised care models and service delivery.

Much existing research has concentrated on diagnostic co-morbidity of ASC and SSD; however, more recently research has found the conditions co-occur at the trait level (Kincaidet al., 2017). This is important given that ASC and SSD are both hypothesised to exist on an extended continuum, with traits and symptoms varying from low, to mid, to potentially diagnostic levels (Constantino \& Todd, 2003; Johns \& van Os, 2001). This means that autism traits which do not reach diagnostic levels may still be clinically important in individuals with SSD, and it is therefore important that the potential impact of traits along the full range of this scale are understood. Autism traits have been found to be stable in SSD, and remain at the same levels regardless of remission of psychotic symptoms (in comparison to depression, for example, where remission from depression also leads to remission from the autism-like traits; Matsuo et al., 2015). This is important as it implies that the high levels of autism traits found in SSD populations are not merely the result of current symptoms of psychosis. There is currently a lack of evidence regarding the clinical impact of autism traits in individuals with SSD. Current treatments for SSD are relatively effective with regards to positive symptoms, however longstanding challenges remain; in particular, patients with SSD often have enduring difficulties with functional recovery and quality of life. It is important that any role that autism traits may play in functioning and quality of life is examined. This knowledge will inform clinical practice decisions regarding the treatment and management of SSD.

The Autism-Spectrum Quotient (AQ) is a questionnaire which was designed specifically to assess autism traits (as opposed to diagnosis). The AQ also provides general population cut offs for high and very high autism traits, which the authors of the AQ suggest are indicative that an ASC 
diagnosis may be appropriate (Baron-Cohen et al., 2001; Woodbury-Smith et al., 2005). The AQ has been used previously with SSD populations to assess autism traits, finding high levels of autism traits compared to healthy controls (Koelkebeck et al., 2010; Lugnegård et al., 2015; Wouters \& Spek, 2011) and that these traits are associated with regional neurological alterations (Sasamoto et al., 2011).

The primary objective of this study was to examine the hypothesis that autism traits in individuals with psychosis will be significantly associated with clinically relevant variables, including symptoms of psychosis, functioning, and quality of life. Exploratory analyses additionally examined which AQ subscales may contribute to this association. Secondary objectives of the study were to determine optimal cut-offs of the AQ with respect to these clinically relevant variables. The measurement of traits of autism in SSD populations is complex and validated instruments for autism assessment in this population do not currently exist. If measures of autism traits (such as the AQ) are measuring genuine traits then these should correlate with premorbid adjustment. A final objective of our research was therefore to examine the relationship between the AQ and premorbid adjustment.

\section{Methods}

\subsection{Design:}

A cross-sectional design was used, consisting of a single assessment of interviewer and selfreported measures conducted and scored by trained raters who were blind to participants AQ scores. Consent was obtained from all participants. Ethical approval was granted by the UK Health Research Authority's National Research Ethics Service, reference number 13/WM/0213.

\subsection{Sampling:}

Outpatients presenting to Birmingham and Solihull Mental Health Foundation Trust Early Intervention Services (EIS) were invited to take part in the research. EIS provides clinical services for young people presenting in the early stages of psychotic illness. Inclusion criteria were individuals aged 16 years or above, diagnosed by EIS consultant psychiatrists in keeping with ICD10 F20-23, F25-29. Consultant psychiatrists are trained in ICD-10 diagnosis, however no specific structured interview was used. Diagnoses were discussed during clinical team meetings to ensure 
diagnostic consensus. First episode of psychosis was established via discussion with EIS clinical teams to determine that participants had not had any previous episodes of psychosis. Exclusion criteria included a lack of capacity to consent and insufficient English language proficiency. With the exception of past psychotic illness (which would deem a person to not be experiencing a first episode of psychosis), past mental illness was not an exclusion criterion. EIS psychiatrists and carecoordinators were asked to systematically identify any individuals on their caseload who met the inclusion and exclusion criteria. Any individuals who met inclusion and exclusion criteria were then approached and asked if they would like to participate in the study. With the participant's consent their medical records were searched for primary diagnosis, diagnosis of autism, and medication. Participants were not antipsychotic-naïve.

\subsection{Measures:}

Demographic information collected included age, gender, ethnicity, living situation, previous cannabis use, educational attainment, work/education status, parental age at birth, and family history of mental illness or autism.

Psychotic symptoms: The Positive and Negative Syndrome Scale (PANSS; Kay et al., 1987) is a 30-item, well-validated, interviewer-rated measure of Positive, Negative, and General psychotic symptoms occurring over the past week. Higher scores on the PANSS indicate greater symptom severity.

Autism Traits: The Autism-Spectrum Quotient (AQ; Baron-Cohen et al., 2001) is a well-validated 50-item self-report questionnaire. The AQ was designed as a measure of autism traits along the wider autism-spectrum and is commonly employed as a screening instrument prior to ASC diagnosis (Woodbury-Smith et al., 2005). Higher scores on the AQ indicate a higher level of autism traits. Baron-Cohen et al. (2001) suggest that AQ scores of $\geq 32$ indicate that an ASC diagnosis may be appropriate, and Woodbury-Smith et al. (2005) argue that individuals with ASC are unlikely to score below 26. These cut-offs were not used in the present study as a diagnostic tool, but were instead used to define high autism traits $(\geq 26)$ and very high autism traits $(\geq 32)$.

The AQ assesses five domains: a preference for non-social situations or difficulty making friends increase participants' scores on the Social Skill subscale; inflexible or fixed attention, commonly reported in ASC, are assessed on the Attention Switching subscale; a focus on details to the exclusion of the bigger picture, and interest in patterns or numbers increase scores on the Attention to Detail subscale; difficulties or a lack of enjoyment in communicating with others is 
assessed on the Communication subscale; and difficulties in activities which involve an imagination component are assessed on the Imagination subscale. The AQ has previously been used as a measure of autism traits within SSD populations (Koelkebeck et al., 2010; Lugnegård et al., 2015; Wouters \& Spek, 2011).

Functioning and Quality of Life: Current functioning (defined as the past month) was assessed using the interviewer-rated Social and Occupational Functioning Assessment (SOFAS; Goldman et al., 1992) and Personal and Social Performance Scales (PSP; Morosini et al., 2000). Higher scores on both of these scales indicate a better level of personal, social, and role functioning. Current quality of life was measured with the interviewer-rated 21 item Quality of Life Scale (QoL; Heinrichs et al., 1984), which can be broken down into subscales representing Social Functioning, Engagement in Life, and Vocational Functioning (Shtasel et al., 1992). Higher scores on the QoL indicate a better quality of life.

Premorbid Adjustment: The Premorbid Assessment of Functioning Scale (PAS; Cannon-Spoor, Potkin, and Wyatt) was used to assess premorbid functioning from childhood through to illness onset, across five areas of development; sociability and withdrawal, peer relationships, scholastic performance, adaption to school, and social sexual aspects of life. Higher scores on this scale are associated with lower levels of premorbid functioning.

\subsection{Analysis:}

Statistical analyses were conducted using SPSS version 22.0. In order to examine the hypothesis that autism traits in individuals with psychosis will be associated with clinically relevant variables, including symptoms of psychosis, functioning, and quality of life a series of main regression analyses were run. Further exploratory post-hoc analyses then examined the relationship between which AQ subscales may contribute to this association.

First, Mann-Whitney U (for scale variables) and Fishers exact tests (for categorical variables) were used to investigate any potential differences in demographics characteristics between high $(\geq 32)$ and lower $(<32)$ scorers on the AQ. To calculate whether potential confounders should be controlled for, correlations between continuous total AQ score and confounders were examined. Correlations between premorbid adjustment and autism were investigated using bivariate correlations. 
Second, where $\mathrm{p}<0.05$ the confounder was included in the model. Qualification status (no qualification or basic qualification only vs higher level of qualification) was included as a potential confounder for the PANSS models. Qualification status and affective vs non-affective diagnosis were included as potential confounders in the QoL and Functioning (SOFAS and PSP) models.

Type of regression used varied depending on whether assumptions were met or not. Multiple linear regression analyses were used where assumptions were met (Total QoL, Engagement QoL, PANSS General). If assumptions of normality were not met (based on a visual inspection of frequency distributions of outcomes, the Kolmogorov-Smirnov test, and normality of residuals), data were transformed using log transformations (PSP and PANSS Total) and multiple linear regression analyses were used. If transformation was not possible (Social QoL, Vocational QoL, SOFAS, PANSS Negative, and PANSS Positive) the variable was categorised into quartiles and multinomial logistic regression analyses were used with the $1^{\text {st }}$ quartile $(\mathrm{Q} 1)$ used as the reference category. Variance inflation factors showed acceptably low levels of multicollinearity between AQ subscales. In total, 10 main regressions were run (one for each outcome). A Bonferroni corrected alpha cut-off of $0.005(0.05 / 10)$ was used to correct for inflation of the false positive rate due to multiple comparisons. Further exploratory post-hoc analyses were run without this conservative correction (alpha $=0.05)$.

Third, to investigate the predictive value of AQ scores for the severity of psychotic symptoms, psychosocial functioning and quality of life, without a priori cut-offs, ROC-curves were calculated to evaluate the prognostic ability of AQ at different cut-offs. This allowed for quantification of the association between the AQ and the outcome across different AQ levels, giving insight into the level at which the predictor becomes indicative of a clinical problem (i.e. is this only at levels of very high autism traits of $\geq 32$ ?). Optimal prediction of the outcome was used as a criterion to select the most relevant AQ cut-off. This approach was chosen because it allowed for the identification of an optimal AQ score i.e. the AQ with optimal true positive rate, or sensitivity (SENS), and false positive rate, or specificity (SPEC) for insight into the general prognostic value of AQ score for poor outcome.

In order to to identify the factors associated with the poorest functioning and most severe symptoms using ROC analyses, the outcomes were coded accordingly. The highest 25\% (1) of PANSS scores (positive, negative, general and total) were compared to the remaining $75 \%$ (0) of PANSS scores. The lowest 25\% (1) of SOFAS scores, QLS scores (total, social and engagement) and PSP scores were compared to the remaining 75\% (0) of SOFAS, QLS and PSP scores. QLS vocational was not included because of a lack of variation in the scores in this sample. After 
calculation of the ROC-curves, the non-parametric area under the curve (AUC) was investigated. If the AUC was significantly different from 0.5 (=chance level prediction), the curve's SENS and SPEC coordinates were inspected to find the AQ score with the most optimal SENS/SPEC balance, which was selected based on the highest J-statistic (SENS+SPEC-1; Youden, 1950). Finally, SENS and SPEC were investigated for the AQ cut-offs of $\geq 32$ (defined as very high autism traits in the present study and suggested by Baron-Cohen et al., 2001, as indicative that an autism spectrum diagnosis may be appropriate), and <26 (suggested by Woodbury-Smith et al., 2005, as indicative that an autism spectrum diagnosis is highly unlikely).

\section{Results}

\subsection{Demographic Information and premorbid adjustment}

Ninety nine individuals took part in the study, of whom 87 gave complete AQ data. Of these, 59 $(67.8 \%)$ participants were male (as is typical in psychosis populations; Aleman et al., 2003). The mean age of the sample was 25.5 years $(S D=5.0)$. Just over half the sample (47 participants) identified as White British, with other common ethnicities including Asian Pakistani (7 participants), Black Caribbean (5 participants), Mixed White and Black Caribbean (4 participants), Asian Bangladeshi (4 participants), and White Other (4 participants). All other ethnicities were represented by less than $4 \%$ of participants. $41.4 \%$ of the sample had a $1^{\text {st }}$ degree relative with a psychiatric condition including; a schizophrenia spectrum diagnosis (12.1\%), a mood disorder $(23.2 \%)$, an autism spectrum disorder $(8 \%)$, and substance abuse $(1 \%)$. The majority $(93.9 \%)$ of participants were currently being prescribed antipsychotic medication. Demographics of participants are reported in Table 1. No significant differences in demographic characteristics were found between those who reported very high levels of autism traits compared to participants with lower scores. As the main regression analyses use continuous AQ scores as the predictor variable, correlations between potential confounders and AQ score was also examined using a point-biserial correlation. This found significant correlations between the AQ and diagnosis $(r=-0.3, p=0.01)$, and educational attainment $(\mathrm{r}=-0.2, \mathrm{p}=0.04)$. These were therefore included as co-variates in all regression analyses. Autism and AQ data can be seen in Table 2. Four participants had a preexisting diagnosis of autism. Due to the research aim of investigating autism traits across the entire continuum, these participants were included in all analyses. 
Premorbid adjustment, as measured by the PAS, was found to be significantly positively correlated with AQ Total $(\mathrm{r}=0.52, \mathrm{p}<0.0001)$. Significant positive correlations were also found between the PAS and AQ Social ( $r=0.35, \mathrm{p}=0.001)$, Attention Switching $(\mathrm{r}=0.44, \mathrm{p}<0.0001)$, Communication $(\mathrm{r}=0.50, \mathrm{p}<0.0001)$, and Imagination $(\mathrm{r}=0.34, \mathrm{p}=0.001)$ subscales, but not with the Attention to Detail subscale.

Table 1: Demographic Characteristics of participants, and significance of difference between those who reported very high levels of traits on the AQ (defined by a score of 32 or over) compared to participants with lower scores

\begin{tabular}{|c|c|c|c|c|}
\hline & All & $\begin{array}{l}\text { Score of }<32 \text { on } \\
\text { AQ }(n=79)\end{array}$ & $\begin{array}{l}\text { Score of } \geq 32 \text { on } \\
A Q(n=8)\end{array}$ & $\begin{array}{l}\text { Significance of } \\
\text { difference, } p \text { value }\end{array}$ \\
\hline Age (Mean, SD) & $25.5(5.0)$ & $25.5(5.0)$ & $25.9(6.8)$ & 0.9 \\
\hline Gender ( $\mathrm{n}, \%$ male $)$ & $59(67.7)$ & $53(67.1)$ & $6(75.0)$ & 1 \\
\hline $\begin{array}{l}\text { Medication (n, } \% \text { not drug } \\
\text { naïve) }\end{array}$ & $83(95.4)$ & $76(96.2)$ & $7(87.5)$ & 0.3 \\
\hline $\begin{array}{l}\text { Mother's age when born } \\
\text { (Mean, SD) }\end{array}$ & $25.8(6.8)$ & $25.8(6.8)$ & $26.3(5.3)$ & 0.7 \\
\hline $\begin{array}{l}\text { Father's age when born } \\
\text { (Mean, SD) }\end{array}$ & $28.8(7.6)$ & $28.8(7.6)$ & $29.2(4.1)$ & 0.6 \\
\hline $\begin{array}{l}\text { Cannabis ( } \mathrm{n}, \% \text { previously } \\
\text { smoked) }\end{array}$ & $58(66.7)$ & $53(67.1)$ & $5(62.5)$ & 1 \\
\hline $\begin{array}{l}\text { Age at first smoking } \\
\text { cannabis (Mean, SD) }\end{array}$ & $15.5(2.7)$ & $15.5(2.7)$ & $16.0(2.2)$ & 0.6 \\
\hline $\begin{array}{l}\text { Living situation (n, \% } \\
\text { Living independently) }\end{array}$ & $34(39.1)$ & $32(40.5)$ & $2(25.0)$ & 0.5 \\
\hline $\begin{array}{l}\text { Relationship status ( } \mathrm{n}, \% \\
\text { single/never married) }\end{array}$ & $70(80.5)$ & $63(79.7)$ & $7(87.5)$ & 1 \\
\hline $\begin{array}{l}\text { Educational attainment (n, } \\
\% \text { no qualifications) }\end{array}$ & $8(9.3)$ & $7(9)$ & $1(12.5)$ & 0.6 \\
\hline $\begin{array}{l}\text { Employment status ( } \mathrm{n}, \% \\
\text { not in education or } \\
\text { employment) }\end{array}$ & $49(57.6)$ & $42(54.5)$ & $7(87.5)$ & 0.1 \\
\hline $\begin{array}{l}\text { History of family mental } \\
\text { illness ( } \mathrm{n}, \% \text { positive } \\
\text { family history) }\end{array}$ & $36(41.4)$ & $31(39.2)$ & $5(62.5)$ & 0.3 \\
\hline $\begin{array}{l}\text { Diagnosis (n, \% Affective } \\
\text { diagnosis) }\end{array}$ & $17(19.5)$ & $17(21.5)$ & $0(0)$ & 0.3 \\
\hline
\end{tabular}




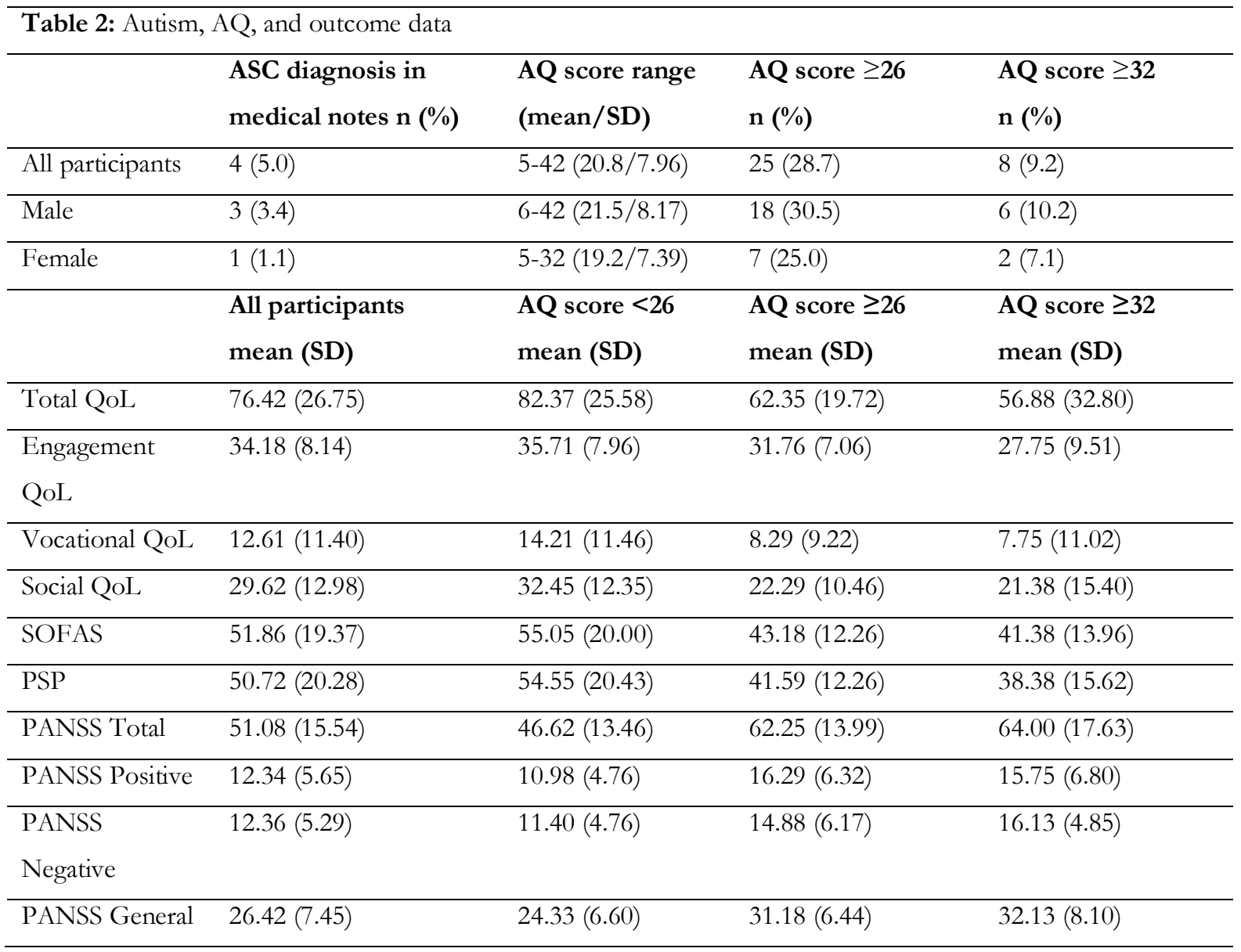

\subsection{The relationship between $A Q$ subscales and symptoms of psychosis, functioning, and quality of life}

Separate multiple linear regression main analyses (Table 3) showed that higher levels of autism traits were significantly associated with lower Total QoL. Exploratory post hoc analyses found that the specific autism traits which showed significant associations were Attention Switching and Imagination. Functioning, as measured by the PSP, was also associated with autism traits, with higher AQ scores predicting lower functioning. Post hoc analyses found the AQ subscale of Imagination showed significant effects. Autism traits were significantly associated with PANSS Total and General subscales in the main regression. Post hoc analyses found that this was driven by the AQ Communication subscale for both PANSS Total and General. The model for Engagement QoL was significant, however this relationship was predominantly driven by Qualification attainment rather than autism traits. 
Table 3: Associations of autism traits with quality of life, current functioning, and current symptoms; multiple linear regression analyses.

\begin{tabular}{|c|c|c|c|c|c|c|c|}
\hline & $\mathbf{R}^{2}$ & $\mathbf{F}$ & $\begin{array}{l}\mathrm{p} \text {-value } \\
\text { for } \mathrm{F}\end{array}$ & $\begin{array}{c}\text { Effect size } \\
\left(\text { Cohen's f }{ }^{2}\right)\end{array}$ & $\beta$ & $95 \% \mathrm{CI}$ & $\begin{array}{c}p \text {-value } \\
\text { for } t\end{array}$ \\
\hline \multicolumn{8}{|l|}{ Quality of life } \\
\hline Total Score & 0.45 & 9.30 & $<0.0001$ & 0.82 & & & \\
\hline AQ Social & & & & & -0.10 & $-3.31,1.29$ & 0.4 \\
\hline AQ Attention Switching & & & & & -0.35 & $-6.83,-1.56$ & 0.002 \\
\hline AQ Attention to Detail & & & & & 0.11 & $-0.39,3.80$ & 0.1 \\
\hline AQ Communication & & & & & 0.04 & $-2.56,3.36$ & 0.8 \\
\hline AQ Imagination & & & & & -0.27 & $-5.89,-0.95$ & 0.007 \\
\hline Qualification attainment & & & & & 0.28 & $5.43,25.95$ & 0.003 \\
\hline Affective diagnosis & & & & & 0.08 & $-6.69,17.92$ & 0.4 \\
\hline Engagement & 0.41 & 7.84 & $<0.0001$ & 0.69 & & & \\
\hline AQ Social & & & & & -0.08 & $-0.98,0.49$ & 0.5 \\
\hline AQ Attention Switching & & & & & -0.22 & $-1.65,0.03$ & 0.06 \\
\hline AQ Attention to Detail & & & & & 0.13 & $-0.17,1.17$ & 0.1 \\
\hline AQ Communication & & & & & -0.05 & $-1.13,0.76$ & 0.7 \\
\hline AQ Imagination & & & & & -0.12 & $-1.26,0.32$ & 0.2 \\
\hline Qualification attainment & & & & & 0.36 & $2.85,9.10$ & $<0.0001$ \\
\hline Affective diagnosis & & & & & 0.15 & $-0.76,7.12$ & 0.1 \\
\hline \multicolumn{8}{|l|}{ Functioning } \\
\hline PSP & 0.37 & 6.73 & $<0.0001$ & 0.59 & & & \\
\hline AQ Social & & & & & 0.08 & $-0.01,0.02$ & 0.5 \\
\hline AQ Attention Switching & & & & & -0.22 & $-0.03,-0.00$ & 0.07 \\
\hline AQ Attention to Detail & & & & & 0.03 & $-0.01,0.02$ & 0.7 \\
\hline AQ Communication & & & & & -0.15 & $-0.03,0.01$ & 0.3 \\
\hline AQ Imagination & & & & & -0.27 & $-0.04,-0.01$ & 0.01 \\
\hline Qualification attainment & & & & & 0.25 & $0.02,0.15$ & 0.01 \\
\hline Affective diagnosis & & & & & 0.12 & $-0.03,0.13$ & 0.2 \\
\hline \multicolumn{8}{|l|}{ Symptoms } \\
\hline PANSS Total & 0.44 & 10.32 & $<0.0001$ & 0.79 & & & \\
\hline AQ Social & & & & & -0.02 & $-0.01,0.01$ & 0.9 \\
\hline AQ Attention Switching & & & & & 0.16 & $-0.00,0.02$ & 0.1 \\
\hline AQ Attention to Detail & & & & & 0.14 & $0.00,0.02$ & 0.1 \\
\hline AQ Communication & & & & & 0.32 & $0.00,0.03$ & 0.02 \\
\hline AQ Imagination & & & & & 0.13 & $-0.00,0.02$ & 0.2 \\
\hline Qualification attainment & & & & & -0.31 & $-0.13,-0.03$ & 0.001 \\
\hline PANSS General & 0.40 & 8.69 & $<0.0001$ & 0.67 & & & \\
\hline AQ Social & & & & & -0.05 & $-0.79,0.53$ & 0.7 \\
\hline AQ Attention Switching & & & & & 0.14 & $-0.25,1.20$ & 0.2 \\
\hline AQ Attention to Detail & & & & & 0.09 & $-0.30,0.90$ & 0.3 \\
\hline AQ Communication & & & & & 0.39 & $0.32,2.01$ & 0.008 \\
\hline AQ Imagination & & & & & 0.10 & $-0.37,1.05$ & 0.3 \\
\hline Qualification attainment & & & & & -0.25 & $-6.47,-0.90$ & 0.01 \\
\hline
\end{tabular}

Five separate multinomial logistic regression main analyses (Table 4) were conducted to investigate associations of AQ subscales with Social and Vocational QoL, the SOFAS, and PANSS Positive and Negative. For these the outcome variable was categorised into quartiles, and the multinomial logistic regression compared the 1st quartile (Q1) to each other quartile, in order to assess how the AQ was associated with the outcome variable. Odds ratios from the main analyses indicated that 
when the contributing AQ subscales were raised Social QoL and SOFAS scores decreased significantly, and PANSS negative scores increased. The multinomial logistic regression analyses for Vocational QoL and PANSS positive were not found to be significant at the corrected p-value of 0.005 .

Exploratory post hoc analyses indicated that on the Social QoL outcome, higher scores on AQ Imagination were associated with lower odds of being in the $2^{\text {nd }}$ and $3^{\text {rd }}$ quartiles compared to Q1, higher scores on AQ Attention to Detail with lower odds of being in the $3^{\text {rd }}$ quartile, higher scores on the AQ Social subscale with lower odds of being in the $4^{\text {th }}$ quartile, and higher scores on the AQ Attention switching subscale with lower odds of being in the $4^{\text {th }}$ quartile. This indicates that higher scores on the AQ subscales of Social, Attention Switching, Attention to Detail, and Imagination may be associated with a reduction in Social QoL. For the SOFAS, higher scores on AQ Attention Switching were associated with lower odds of being in the $3^{\text {rd }}$ and $4^{\text {th }}$ quartile compared to Q1, and higher scores on AQ Imagination were associated with lower odds of being in the $3^{\text {rd }}$ quartile compared to Q1. This indicates that higher scores on the AQ subscales of Attention Switching and Imagination are associated with a reduction in functioning. For the PANSS negative, higher scores on AQ communication were associated with higher odds of being in the $3^{\text {rd }}$ quartile compared to Q1, and higher scores on AQ Imagination were associated with higher odds of being in the $4^{\text {th }}$ quartile compared to Q1. This indicates that higher scores on the AQ subscales of Communication and Imagination may be associated with an increase in negative symptoms. 
Table 4: Associations of autism traits with Quality of Life, current functioning, and current symptoms; Multinomial logistic regressions overall model significance, Nagelkerke's $\mathrm{R}^{2}$, and significantly contributing predictors for each quartile compared to the reference group ( $1^{\text {st }}$ quartile)

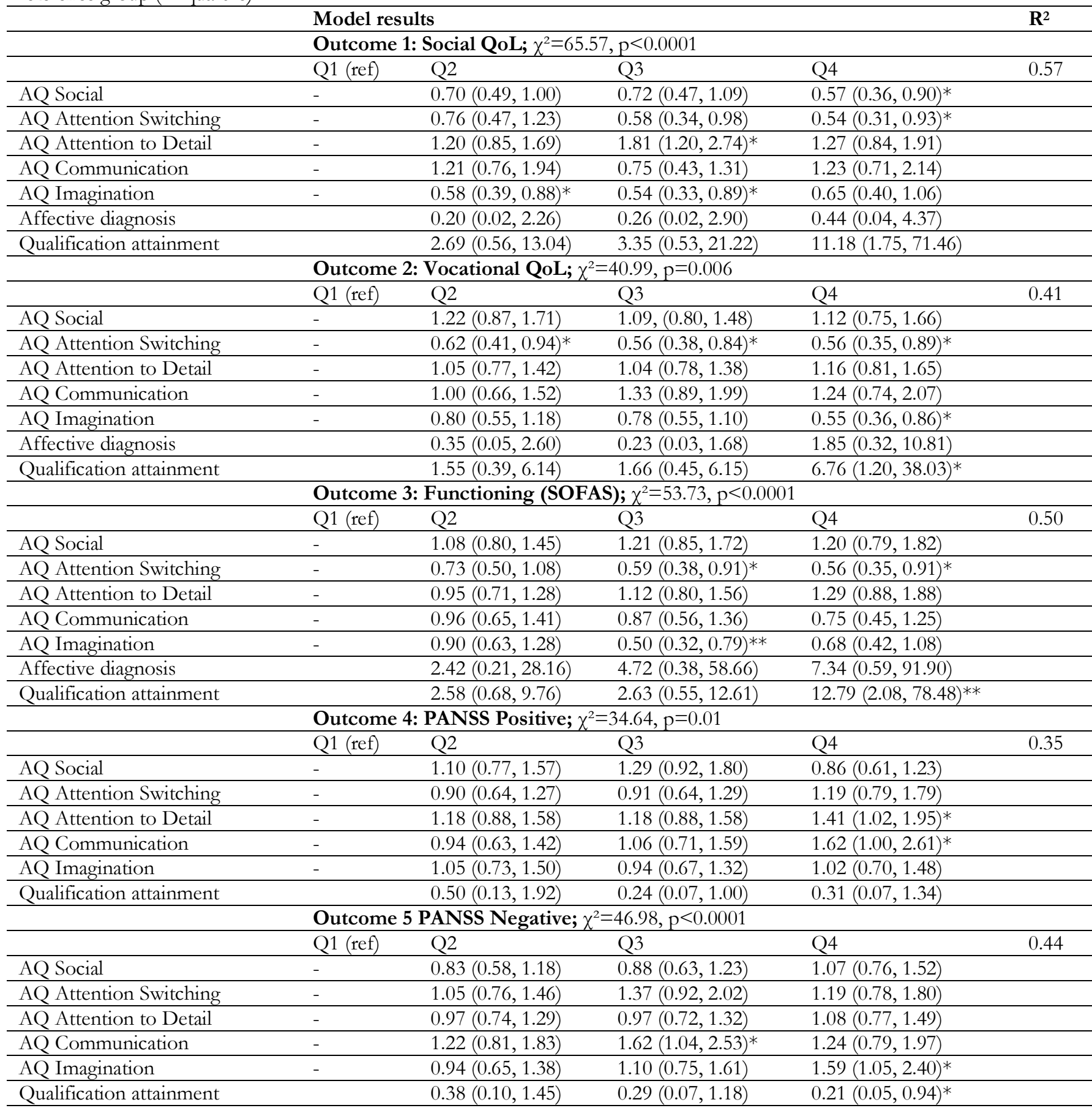

Note: data are OR's with $95 \%$ confidence intervals

*) $\mathrm{p}<0.05$;

**) $\mathrm{p}<0.01$;

Categorical variables were constructed as follows: Social QoL $(2-17=Q 1,19-29=Q 2,30-41=Q 3,42-48=Q 4)$, Vocational QoL $(0-$ $2=\mathrm{Q} 1,3-9=\mathrm{Q} 2,10-24=\mathrm{Q} 3,26-30=\mathrm{Q} 4)$, SOFAS $(21-40=\mathrm{Q} 1,41-45=\mathrm{Q} 2,46-61=\mathrm{Q} 3,63-95=\mathrm{Q} 4)$, PANSS Positive $(7-8=\mathrm{Q} 1 ; 9-$ $10=\mathrm{Q} 2 ; 11-16=\mathrm{Q} 3 ; 17-30=\mathrm{Q} 4), \mathrm{PANSS}$ Negative $(7-8=\mathrm{Q} 1,9-11=\mathrm{Q} 2,12-16=\mathrm{Q} 3,17-31=\mathrm{Q} 4)$. 
ROC-curves were calculated using AQ (range=5-42; $\mathrm{M}=20.76 ; \mathrm{SD}=7.96$ ) as a predictor variable. First, the AUCs for the ROCs with different outcomes were investigated to gain an overall insight of the association of AQ scores with poor outcome. For each ROC analysis conducted, the AUC differed significantly from 0.5 , suggesting that total AQ was predictive of all the outcomes examined. AUC values ranged from 0.72 to 0.85 .

Next, we examined the optimal SENS/SPEC for each outcome. AQ scores between 19.5 and 25.5 were the cut-offs for optimal SENS/SPEC, depending on the outcome of interest. The optimal AQ cut-off for each outcome is shown in Table 5. These observed optimal AQ cut-offs are lower than the published cut-offs of 26 and 32. These cut-offs had better SENS, but SPEC was compromised compared to the cut offs of 26 and 32 . The J-statistic was lower for the cut-offs of 26 and 32 . 
Table 5. Optimal AQ cut-off, cut-off at 26 and cut-off at 32

\begin{tabular}{lrrrr}
\hline Optimal cut off & AQ score & Sensitivity $\%$ & Specificity $\%$ & J-statistic \\
\hline PANSS positive & 25.5 & 59.1 & 81.5 & 0.406 \\
\hline PANSS negative & 19.5 & 87.5 & 60.3 & 0.478 \\
\hline PANSS general & 22.5 & 88.5 & 73.3 & 0.618 \\
\hline PANSS total & 19.5 & 91.3 & 61.9 & 0.532 \\
\hline QLS total & 22.5 & 84.0 & 69.4 & 0.534 \\
\hline QLS social & 23.5 & 76.2 & 72.7 & 0.489 \\
\hline QLS engagement & 23.5 & 72.0 & 74.2 & 0.462 \\
\hline SOFAS & 19.5 & 80.8 & 59.0 & 0.398 \\
\hline PSP & 19.5 & 79.2 & 57.1 & 0.363 \\
\hline
\end{tabular}

\begin{tabular}{lrrrr}
\hline Cut off at 26 & AQ score & Sensitivity $\mathbf{0}$ & Specificity $\mathbf{0}$ & J-statistic \\
\hline PANSS positive & 26.5 & 54.5 & 84.6 & 0.392 \\
\hline PANSS negative & 26.5 & 41.7 & 81.0 & 0.226 \\
\hline PANSS general & 26.5 & 53.8 & 86.7 & 0.405 \\
\hline PANSS total & 26.5 & 52.2 & 84.1 & 0.363 \\
\hline QLS total & 26.5 & 52.0 & 85.5 & 0.375 \\
\hline QLS social & 26.5 & 47.6 & 81.8 & 0.294 \\
\hline QLS engagement & 26.5 & 48.0 & 83.9 & 0.319 \\
\hline SOFAS & 26.5 & 42.3 & 82.0 & 0.243 \\
\hline PSP & 26.5 & 45.8 & 82.5 & 0.284 \\
\hline
\end{tabular}

\begin{tabular}{lrrrr}
\hline Cut off at 32 & AQ score & Sensitivity $\mathbf{0}$ & Specificity $\mathbf{0}$ & J-statistic \\
\hline PANSS positive & 32.5 & 13.6 & 96.9 & 0.106 \\
\hline PANSS negative & 32.5 & 12.5 & 96.8 & 0.093 \\
\hline PANSS general & 32.5 & 11.5 & 96.7 & 0.082 \\
\hline PANSS total & 32.5 & 13.0 & 96.8 & 0.099 \\
\hline QLS total & 32.5 & 16.0 & 98.4 & 0.144 \\
\hline QLS social & 32.5 & 19.0 & 98.5 & 0.175 \\
\hline QLS engagement & 32.5 & 16.0 & 98.4 & 0.144 \\
\hline SOFAS & 32.5 & 11.5 & 96.7 & 0.083 \\
\hline PSP & 32.5 & 16.7 & 98.4 & 0.151 \\
\hline
\end{tabular}

\section{Discussion}

This is the first study to examine the association between traits of autism with current functioning, quality of life, and psychotic symptoms in a psychosis population. Higher levels of autism traits were found to be associated with lower quality of life, lower functioning, and higher levels of current psychotic symptoms. Exploratory post hoc analyses found that this association was particularly strong for the AQ Attention Switching and Imagination subscales for quality of life 
and functioning, and for the AQ Communication subscale for current total and negative psychotic symptoms. A ROC analysis indicated that autism traits which fall below potentially diagnostic levels may still be clinically important in relation to their impact on symptoms of psychosis, functioning, and quality of life. It is also of note that $8.1 \%$ participants had scores of $\geq 32$ (indicative of very high autism traits; Baron-Cohen et al., 2001) with just over a quarter of participants scoring $\geq 26$ (indicative of high autism traits; Woodbury-Smith et al., 2005). This compares to scores of $\geq 32$ in just $2 \%$ of the general population (Baron-Cohen et al., 2001).

Previously ASC have been shown to be related to increased risk factors for psychopathology (including psychosis), including increased exposure to negative life events (e.g. Maïano et al., 2015), poorer coping styles (Mazefsky et al., 2014), reduced executive functioning (Merchán-Naranjo et al., 2016), and reduced social support networks (Chamberlain et al., 2007). In non-clinical populations, autism traits have been linked to lower quality of life, mediated by less successful coping styles (Pisula et al., 2015). Individuals with increased autism traits also report more loneliness (Jobe \& White, 2007), and higher rates of adverse life events (Kunihira et al., 2006). The AQ subscales of Attention Switching, Communication, and Imagination were found to drive many of the associations between the AQ and the clinically significant variables. Attention switching difficulties are part of the core diagnostic criteria for ASC, and are also common in SSD. In the general population, attention switching skills are hypothesised to underlie problem solving abilities (Byrne \& Murray, 2005), suggesting that this may be a potential mediator between autism traits and psychosocial functioning in first-episode psychosis populations. Communication difficulties are also found in both ASC and SSD populations. Alexithymia describes a difficulty in identifying and communicating emotions, and is commonly found in ASC (Bird \& Cook, 2013). In individuals with high autism traits, higher levels of alexithymia could potentially prevent early help-seeking, leading to under-reporting of psychopathology, increased duration of untreated illness, and more severe clinical outcomes.

The ROC analyses showed that optimal AQ cut-offs for poor clinical outcome were lower than the cut-offs of 32 and 26 suggested as potentially indicative of ASC in the general population (Baron-Cohen et al., 2001; Woodbury-Smith et al., 2005). Optimal cut-offs varied from 19.5 to 25.5. It is important that this finding is not taken to suggest that a lower AQ score may be indicative of autism in FEP populations. Rather, this is suggestive that in psychosis populations, levels of autism traits which fall below potentially diagnostic levels may still be highly clinically relevant in terms of their potential relationship with current symptoms of psychosis, quality of life, and functioning. This may be particularly the case for the variables where the lowest optimal cut offs 
were found (PANSS Negative, PANSS Total, and functional outcomes). It is important to note, however, that this is exploratory initial work and there is need for further confirmation and validation.

Premorbid adjustment was found to be significantly positively correlated with the AQ Total, meaning that higher autism traits were associated with lower premorbid adjustment. This is suggestive that the AQ was measuring genuine long term autism-like traits, as opposed to capturing a state-like refection of current psychotic illness.

A number of models which may explain the co-occurrence of ASC and SSD have been proposed (Chisholm et al., 2015; Crespi \& Badcock, 2008; Kraemer, 1995; Krueger \& Markon, 2006).

These include the diametric model, which hypothesises that ASC and SSD originate from opposing alterations to a common risk factor, and are therefore related in a reciprocal manner (Crespi \& Badcock, 2008). The diametric model suggests that when individuals experience high levels of both psychotic-like and autism-like traits, behaviour, symptoms, and functioning will be modulated diametrically back towards normality. Whilst counterintuitive, there is emerging neurological (Abu-Akel et al., 2017; Ciaramidaro et al., 2014) and cognitive (Abu-Akel et al., 2015) research which supports this theory, particularly within non-clinical populations. The current study found that in a population with psychosis (all of whom can therefore be considered to be 'high psychosis'), higher levels of autism traits were not associated with an ameliorating impact on symptoms and functioning, and were instead related to a lower quality of life, lower functioning, and higher levels of psychotic symptoms. This suggests that within clinical psychosis populations, the diametric model may be less applicable than in non-clinical populations. Much of the existing research into the diametric model has investigated the impact of autism and psychosis traits on theory of mind and mentalising ability (Abu-Akel et al., 2017; Abu-Akel et al., 2015; Ciaramidaro et al., 2014). It is possible that the diametric impact of autism and psychosis is restricted to these areas, although recent research suggests this is unlikely, finding that in neurotypical adults there may be a diametric effect of autism and psychosis traits on atypical responses to salient information (Abu-Akel et al., 2016). It is also possible that the diametric model may be more applicable to certain subgroups of diagnoses than others, and further research is needed to elucidate this. As the diametric model hypotheses an ameliorating effect of autism traits on psychosis and vice versa, it is possible that individuals with high levels of autism traits and psychosis traits would not be found in clinical samples, and would only be found in a general population sample. This is a potential explanation for the lack of supporting evidence for the diametric model in the current sample, however, some of the study participants 
did report relatively high levels of autism traits, and 4 individuals had a pre-existing diagnosis of autism. If the diametric model is correct, it seems likely that within the present sample, individuals reporting higher levels of autism traits should have shown some level of amelioration in their psychosis symptoms, functioning, or quality of life, even if this amelioration effect was not strong. The observation that the opposite pattern occurred, with those with higher autism traits reporting higher psychosis symptoms, and lower functioning and quality of life, suggests that the diametric model does not apply to the current sample.

The study has a number of limitations. The AQ was used rather than a diagnostic measure such as the Autism Diagnostic Interview-Revised (ADI-R) (Rutter et al, 2003) or the Autism Diagnostic Observation Schedule (ADOS) (Lord et al., 2000). This was due to the fact that whilst the ADI-R and ADOS are considered to be gold standard assessment tools for the diagnosis of autism, the focus of this study was on traits of autism rather than diagnosis. Neither the ADI-R nor the ADOS is specifically designed to measure traits of autism, whereas the AQ was designed with this in mind. The AQ has previously been used as a measure of autism traits within SSD populations (Koelkebeck et al., 2010; Lugnegård et al., 2015; Wouters \& Spek, 2011), however, the measurement of traits of autism in SSD populations is complex and validated instruments for autism assessment in this population do not currently exist. Similarly, validated measures do not exist for the measurement of negative symptoms of psychosis in ASC populations. The relationship between autism traits and psychosis is not yet fully understood and various models which might explain this co-occurrence have been proposed (Chisholm et al., 2015). Many symptoms or experiences which are found in one disorder are also found in the other (for example, the attention switching difficulties which are part of the core ASC criteria and are also found at high rates in SSD), meaning that instruments which assess autism traits in the general population may be less accurate in SSD populations. It is important that researchers take this into consideration. Matsuo et al.'s (2015) finding that autism traits are stable in SSD populations, alongside our finding that the AQ correlated with premorbid adjustment, does however, suggest that autism traits found in SSD populations can be considered genuine traits rather than as merely reflective of current symptoms of psychosis (this can be contrasted with autism traits in depression, where remission from depression led to individuals no longer experiencing the autism-like traits; Matsuo et al., 2015). Until the association between ASC and SSD is better understood, researchers (particularly those conducting cross-sectional research) and clinicians must be careful not to allow negative symptoms of psychosis to mask potential autism traits, which may be less apparent than autism of a diagnosable level. It is possible that autism traits may be misdiagnosed or mislabelled as psychotic symptoms by clinicians on initial presentation to services. The best way to tease this 
apart would be to either to know about previous trait presentation or to assess the trajectory over the treatment period and see if AQ scores decline with psychosis symptom resolution (which they should not if they are traits). For the current research, it is important to note that the AQ assessment is a self-reported response to clear 'trait' questions, and is not externally assessed. In relation to this, we decided against controlling for PANSS symptoms in the current analysis (for the QoL and Functioning outcomes). This was due to the potential for interviewer ratings on the PANSS to be reflecting autism traits rather than genuine symptoms of psychosis, and therefore to mask the impact of any genuine autism traits.

It is possible that the associations found in the present research between the AQ and the variables of interest may be mediated by other variables such as negative symptoms of psychosis, depression, lower IQ, education, or medication. The current sample is not large enough to investigate mediation via path analysis however this would be interesting to pursue in future studies. In addition, future studies should take into account duration of untreated psychosis, as this has been robustly associated with psychotic symptoms, functioning and quality of life (Marshall, Lewis, and Lockwood, 2005). The sampling method used in the study recruitment was not random, but relied on clinical care teams introducing the study to service users, which may have biased the sample of participants. The use of self-report instruments in psychosis populations is relatively common, however, 'informant' information from family, friends, or clinical services on traits of autism in participants may aid the reliability of the assessment and should be considered for future research. Future research could also investigate the clinical impact of autism traits on particular subgroups of people with psychotic disorder, including schizophrenia or non-affective psychosis, childhood onset schizophrenia, and deficit schizophrenia, as some subgroups may have a stronger association with autism than others (Padgett et al, 2010; Sprong et al., 2008). Additionally, as a cross-sectional study, speculation on causality in the present study is limited, and further prospective research is needed.

It is important that psychosis services have built in systems to adequately address the needs of individuals with higher autism traits. These could include the screening of all newly referred individuals for autism traits, and adapted interventions for high trait individuals. For example, simple structural elements such as planning, maintaining consistency and structure should be considered. In addition, communication (verbal and written) and checking of understanding may be of particular importance in individuals with high ASC traits. Clinicians may need to be aware of the need for 'sameness', routine, and structure in interactions with individuals with high autism traits. It is important that clinical teams support individuals with their autism traits to full recovery 
from psychotic symptoms, but recognise autism traits rather than attributing all difficulties to psychosis. As risk factors for psychosis and other psychopathology is increased in those with high levels of autism traits (Jobe \& White, 2007; Kunihira et al., 2006; Maïano et al., 2015), alongside reduced coping skills (Pisula et al., 2015), all clinical services should be aware and able to tailor their approach and treatment appropriately. An example of this can be seen in the autism clinic at Orygen Youth Health, Australia, which recognised the need to expand autism services within the public mental health sector during times of transition (such as adolescence and young adulthood) when the risk of a person with an ASC developing a comorbid mental health problem increases. The role of the autism clinic thus far has included providing comprehensive multidisciplinary diagnostic assessments for ASCs, and provision of both primary and secondary consultations relating to ASC within the other clinical programs of Orygen Youth Health. Another key component of the service has been capacity building and training of staff within Orygen Youth Health. Future research should also investigate whether recognised interventions, such as cognitive-behavioural therapy for psychosis, can be adapted for individuals with a high level of autism traits. Investigating autism diagnoses and traits in individuals who may be at risk for psychosis may also be beneficial for understanding how the disorders interact to exacerbate or ameliorate each other as psychosis develops.

\section{Acknowledgement}

We would like to acknowledge the Birmingham and Solihull Mental Health Foundation Trust Early Intervention Services who aided with the recruitment of this study, and to thank the individuals from the Early Intervention Services who took part in the project as participants. This work was supported by Birmingham and Solihull Mental Health Foundation Trust. AL is supported by an NHMRC Career Development Fellowship (\#1148793).

\section{References:}

Abu-Akel, A., Apperly, I. A., Wood, S. J., \& Hansen, P. C., 2015. Autism and Psychosis Traits Diametrically Modulate the Right Temporo-parietal Junction. European Psychiatry, 30.

Abu-Akel, A., Apperly, I. A., Wood, S. J., Hansen, P. C., \& Mevorach, C., 2016. Autism tendencies and psychosis proneness interactively modulate saliency cost. Schizophrenia Bulletin, 43(1), 142-151.

Abu-Akel, A. M., Apperly, I. A., Wood, S. J., \& Hansen, P. C., 2017. Autism and psychosis expressions diametrically modulate the right temporoparietal junction. Social neuroscience, 12(5), 506-518. 
Aleman, A., Kahn, R. S., \& Selten, J.-P., 2003. Sex differences in the risk of schizophrenia: evidence from meta-analysis. Archives of general psychiatry, 60(6), 565-571.

Baron-Cohen, S., Wheelwright, S., Skinner, R., Martin, J., \& Clubley, E., 2001. The Autism-Spectrum Quotient (AQ): Evidence from Asperger Syndrome/High-Functioning Autism, Malesand Females, Scientists and Mathematicians. Journal of autism and developmental disorders, 31(1), 517.

Bird, G., \& Cook, R., 2013. Mixed emotions: the contribution of alexithymia to the emotional symptoms of autism. Translational psychiatry, 3(7), e285.

Byrne, R. M., \& Murray, M. A., 2005. Attention and working memory in insight problem-solving. Paper presented at the Proceedings of the Cognitive Science Society.

Cannon-Spoor, H.E., Potkin, S.G., and Wyatt, R.J., 1982. Measurement of Premorbid Adjustment in Chronic Schizophrenia. Schizophrenia Bulletin. 8(3). 470-484.

Carroll, L. S., \& Owen, M. J., 2009. Genetic overlap between autism, schizophrenia and bipolar disorder. Genome Med, 1(10), 102.

Chamberlain, B., Kasari, C., \& Rotheram-Fuller, E., 2007. Involvement or isolation? The social networks of children with autism in regular classrooms. Journal of autism and developmental disorders, 37(2), 230-242.

Cheung, C., Yu, K., Fung, G., Leung, M., Wong, C., Li, Q., Sham, P., Chua, S., McAlonan, G., 2010. Autistic disorders and schizophrenia: related or remote? An anatomical likelihood estimation. PLoS One, 5(8), e12233.

Chisholm, K., Lin, A., Abu-Akel, A., \& Wood, S. J., 2015. The association between autism and schizophrenia spectrum disorders: a review of eight alternate models of co-occurrence. Neuroscience \& Biobehavioral Reviews, 55, 173-183.

Ciaramidaro, A., Bölte, S., Schlitt, S., Hainz, D., Poustka, F., Weber, B., Bara, B.G., Freitag, C., Walter, H., 2014. Schizophrenia and autism as contrasting minds: neural evidence for the hypo-hyperintentionality hypothesis. Schizophrenia Bulletin, 41(1), 171-179.

Constantino, J. N., \& Todd, R. D., 2003. Autistic traits in the general population: a twin study. Archives of general psychiatry, 60(5), 524-530.

Crespi, B., \& Badcock, C., 2008. Psychosis and autism as diametrical disorders of the social brain. Behavioral and Brain Sciences, 31(03), 241-261.

Davidson, C., Greenwood, N., Stansfield, A., \& Wright, S., 2014. Prevalence of Asperger syndrome among patients of an Early Intervention in Psychosis team. Early Intervention in Psychiatry, 8(2), 138-146. doi:10.1111/eip.12039

Goldman, H. H., Skodol, A. E., \& Lave, T., 1992. Revising axis V for DSM-IV: a review of measures of social functioning. The American Journal of Psychiatry, 149, 1148-1156.

Heinrichs, D. W., Hanlon, T. E., \& Carpenter, W. T., 1984. The Quality of Life Scale: an instrument for rating the schizophrenic deficit syndrome. Schizophrenia Bulletin, 10(3), 388-398. doi:10.1093/schbul/10.3.388

Hofvander, B., Delorme, R., Chaste, P., Nydén, A., Wentz, E., Ståhlberg, O., Herbrecht, E., Stopin, A., Anckarsäter, H., Gillberg, C., Råstam, M., \& Leboyer, M., 2009. Psychiatric and psychosocial problems in adults with normal-intelligence autism spectrum disorders. BMC psychiatry, 9(1), 1.

Jobe, L. E., \& White, S. W., 2007. Loneliness, social relationships, and a broader autism phenotype in college students. Personality and Individual Differences, 42(8), 1479-1489.

Johns, L. C., \& van Os, J., 2001. The continuity of psychotic experiences in the general population. Clinical Psychology Review, 21(8), 1125-1141.

Kay, S. R., Flszbein, A., \& Opfer, L. A., 1987. The positive and negative syndrome scale (PANSS) for schizophrenia. Schizophrenia Bulletin, 13(2), 261-276.

Kincaid, D. L., Doris, M., Shannon, C., \& Mulholland, C., 2017. What is the prevalence of Autism Spectrum Disorder and ASD traits in psychosis? A systematic review. Psychiatry Research.

Koelkebeck, K., Pedersen, A., Suslow, T., Kueppers, K. A., Arolt, V., \& Ohrmann, P., 2010. Theory of Mind in first-episode schizophrenia patients: correlations with cognition and personality traits. Schizophrenia Research, 119(1-3), 115-123.

Kraemer, H. C., 1995. Statistical issues in assessing comorbidity. Statistics in Medicine, 14(8), 721-733.

Krueger, R. F., \& Markon, K. E., 2006. Reinterpreting comorbidity: A model-based approach to understanding and classifying psychopathology. Annual review of clinical psychology, 2, 111. 
Kunihira, Y., Senju, A., Dairoku, H., Wakabayashi, A., \& Hasegawa, T., 2006. 'Autistic'traits in nonautistic Japanese populations: relationships with personality traits and cognitive ability. Journal of autism and developmental disorders, 36(4), 553-566.

Lord, C., Risi, S., Lambrecht, L., Cook, E. H., Leventhal, B. L., DiLavore, P. C., Pickles, A., Rutter, M., 2000. The Autism Diagnostic Observation Schedule-Generic: A standard measure of social and communication deficits associated with the spectrum of autism. Journal of autism and developmental disorders, 30(3), 205-223.

Lugnegård, T., Hallerbäck, M. U., \& Gillberg, C., 2015. Asperger syndrome and schizophrenia: Overlap of self-reported autistic traits using the Autism-spectrum Quotient (AQ). Nordic journal of psychiatry, 69(4), 268-274.

Maïano, C., Normand, C. L., Salvas, M. C., Moullec, G., \& Aimé, A., 2015. Prevalence of School Bullying Among Youth with Autism Spectrum Disorders: A Systematic Review and Meta-Analysis. Autism Research.

Matsuo, J., Kamio, Y., Takahashi, H., Ota, M., Teraishi, T., Hori, H., Nagashima, A., Takei, R., Higuchi, T., Motohashi, N., Kunugi, H., 2015. Autistic-Like Traits in Adult Patients with Mood Disorders and Schizophrenia. PLoS One, 10(4). doi:10.1371/journal.pone.0122711

Marshall, M, Lewis, S., and Lockwood, A., 2005. Association Between Duration of Untreated Psychosis and Outcome in Cohorts of First-Episode Patients: A Systematic Review. Arch Gen Psychiatry. 62(9). 975-983.

Mazefsky, C. A., Borue, X., Day, T. N., \& Minshew, N. J., 2014. Emotion Regulation Patterns in Adolescents With High-Functioning Autism Spectrum Disorder: Comparison to Typically Developing Adolescents and Association With Psychiatric Symptoms. Autism Research, 7(3), 344-354.

Merchán-Naranjo, J., Boada, L., del Rey-Mejías, Á., Mayoral, M., Llorente, C., Arango, C., \& Parellada, M., 2016. Executive function is affected in autism spectrum disorder, but does not correlate with intelligence. Revista de Psiquiatría y Salud Mental (English Edition), 9(1), 39-50.

Morosini, P., Magliano, L., Brambilla, L., Ugolini, S., \& Pioli, R., 2000. Development, reliability and acceptability of a new version of the DSM-IV Social and Occupational Functioning Assessment Scale (SOFAS) to assess routine social funtioning. Acta Psychiatrica Scandinavica, 101(4), 323329.

Padgett, F. E., Miltsiou, E., \& Tiffin, P. A., 2010. The co-occurrence of nonaffective psychosis and the pervasive developmental disorders: A systematic review. Journal of Intellectual and Developmental Disability, 35(3), 187-198.

Pisula, E., Danielewicz, D., Kawa, R., \& Pisula, W., 2015. Autism spectrum quotient, coping with stress and quality of life in a non-clinical sample-an exploratory report. Health and quality of life outcomes, 13(1), 173.

Rutter, M., Le Couteur, A., \& Lord, C., 2003. Autism diagnostic interview-revised. Los Angeles, CA: Western Psychological Services, 29, 30.

Sasamoto, A., Miyata, J., Hirao, K., Fujiwara, H., Kawada, R., Fujimoto, S., Fujimoto, S., Tanaka, Y., Kubota, M., Sawamoto, N., Fukuyama, H., Takahashi, H., \& Murai, T., 2011. Social impairment in schizophrenia revealed by Autism-Spectrum Quotient correlated with gray matter reduction. Social neuroscience, 6(5-6), 548-558.

Shtasel, D., Gur, R., Gallacher, F., Heimberg, C., Cannon, T., \& Gur, R., 1992. Phenomenology and functioning in first-episode schizophrenia. Schizophrenia Bulletin, 18, 449-462.

Sprong, M., Becker, H., Schothorst, P., Swaab, H., Ziermans, T., Dingemans, P., Linszen, D., van Engeland, H., 2008. Pathways to psychosis: A comparison of the pervasive developmental disorder subtype Multiple Complex Developmental Disorder and the "At Risk Mental State". Schizophrenia Research, 99(1-3), 38-47.

Woodbury-Smith, M. R., Robinson, J., Wheelwright, S., \& Baron-Cohen, S., 2005. Screening adults for Asperger syndrome using the AQ: A preliminary study of its diagnostic validity in clinical practice. Journal of autism and developmental disorders, 35(3), 331-335.

Wouters, S. G. M., \& Spek, A. A., 2011. The use of the Autism-spectrum Quotient in differentiating highfunctioning adults with autism, adults with schizophrenia and a neurotypical adult control group. Research in Autism Spectrum Disorders, 5(3), 1169-1175. doi:10.1016/j.rasd.2011.01.002 